\title{
EFEITO DA APLICAÇÃO DE PLASMA NÃO-TÉRMICO ATMOSFÉRICO NO REPARO DE CERÂMICAS CAD/CAM DE MATRIZ RESINOSA COM RESINA COMPOSTA
}

\author{
EFFECT OF NON-THERMAL ATMOSPHERIC PLASMA APPLICATION ON THE \\ RESIN COMPOSITE REPAIR OF RESIN MATRIX CAD/CAM CERAMICS
}

\author{
Beatriz Mendonça Curvello"; Eduardo Fernandes de Castro²; Veber Luiz Bomfim de Azevedo³; Adriana da Fonte Porto \\ Carreiro4; Simone Duarte ${ }^{5}$; Marcelo Giannini ${ }^{6}$ \\ 1 - Mestre em Clínica Odontológica - Dentística Restauradora, Departamento de Odontologia Restauradora, Faculdade de Odontologia de \\ Piracicaba, Universidade Estadual de Campinas, Piracicaba, SP, Brasil. \\ 2- Doutorando em Clínica Odontológica - Dentística Restauradora, Departamento de Odontologia Restauradora, Faculdade de Odontologia de \\ Piracicaba, Universidade Estadual de Campinas, Piracicaba, SP, Brasil. \\ 3 - Professor de Prótese Dentária, Faculdade de Ilhéus - Cesupi, Ilhéus, BA, Brasil. \\ 4 - Professora Associada de Prótese Dentária e Implantodontia, Departamento de Odontologia, Universidade Federal do Rio Grande do Norte, \\ Natal, Brasil. \\ 5 - Professora Associada, Departamento de Ciência Básica e Biologia Craniofacial, Indiana University-Purdue University Indianapolis, Indianapolis, EUA. \\ 6 - Professor Associado de Dentística Restauradora, Departamento de Odontologia Restauradora, Faculdade de Odontologia de Piracicaba, \\ Universidade Estadual de Campinas, Piracicaba, SP, Brasil.
}

\section{RESUMO}

O objetivo deste trabalho foi avaliar o efeito do plasma atmosférico não-térmico (PANT), sozinho ou associado ao jateamento ou adesivo, e do tempo de armazenamento em água na resistência de união por cisalhamento (RUC) do reparo de cerâmicas CAD/CAM de matriz resinosa (CMR) com resina composta. Amostras de $14 \times 7 \times 1 \mathrm{~mm}$ de três CMRs, Enamic (VITA Zahnfabrik), Cerasmart (GC Corp.) e Lava Ultimate (3M Oral Care), foram obtidas e submetidas ao envelhecimento artificial (EQ-UV, Equilam) por 300 horas. Os seguintes tratamentos de superfície foram realizados: (1- Controle) jateamento + silano + adesivo; (2) PANT; (3) PANT + adesivo; (4) jateamento + PANT. Cilindros de resina composta (Spectra Smart, Dentsply Sirona, $1,5 \mathrm{~mm}$ diâmetro e altura) foram aderidos às superfícies tratadas e a RUC foi avaliada em uma máquina de ensaio universal (EZ
Test, Shimadzu) após 24 horas ou 1 ano de imersão em água, a $37^{\circ} \mathrm{C}(\mathrm{n}=10)$. No geral, o tratamento controle obteve os melhores resultados de RUC, comparado aos grupos tratados com PANT. Houve redução da RUC após 1 ano de imersão em água para a maioria dos grupos, entre eles para o controle do Enamic e Lava Ultimate, enquanto Cerasmart não mostrou redução. $\mathrm{O}$ tratamento com PANT, sozinho ou associado a outro tratamento, não foi capaz de aumentar a RUC do reparo das CMRs com resina composta. O tratamento controle parece ser o melhor método de reparo das CMRs, principalmente considerando-se a longevidade do tratamento.

Palavras-chave: CAD-CAM; Reparação de restauração dentária; Resistência ao cisalhamento; Gases em plasma; Resinas compostas; Porcelana dentária.

\section{INTRODUÇÃO}

Com a evolução das tecnologias digitais CAD/CAM (desenho assistido por computador / manufatura assistida por computador, do inglês - computer-aided design / computer-aided manufacturing) em Odontologia, diversos materiais restauradores foram lançados no mercado. Apesar da grande procura por restaurações indiretas cerâmicas na última década, devido à disseminação da técnica e evolução das propriedades mecânicas e ópticas, algumas limitações das cerâmicas CAD/CAM abriram espaço para o surgimento de outros materiais para fresagem ${ }^{1,2}$.

A alta dureza das cerâmicas CAD/CAM resulta em grande desgaste das brocas de fresagem. Além disso, etapas laboratoriais adicionais como a necessidade de queima de cristalização ou maquiagem e aplicação de glaze pós-fresagem aumentam a complexidade da manufatura deste tipo de material protético ${ }^{3-5}$. Visando facilitar e agilizar o processamento de materiais CAD/ CAM, empresas desenvolveram materiais híbridos com uma composição mista de matriz inorgânica e matriz orgânica. Estes materiais foram recentemente classificados como cerâmicas de matriz resinosa (CMRs) ${ }^{6}$ e apresentam uma manufatura mais simples, rápida e de menor desgaste às brocas, comparados às cerâmicas $\mathrm{CAD} / \mathrm{CAM}^{3-5}$.

O uso crescente das CMRs fez com que diversos estudos avaliassem a adesão de cimentos resinosos para cimentação adesiva 
dos mesmos ${ }^{7-13}$. Entretanto, no ambiente oral estas restaurações podem sofrer danos com o tempo e necessitar de reparos, e esse cenário clínico também deve ser estudado ${ }^{14-24}$. Além disso, a natureza híbrida desses materiais, faz com que a adesão aos mesmos seja desafiadora, pois o tratamento ideal para materiais com conteúdo resinoso e partículas de vidro, pode não ser o melhor para materiais de origem puramente inorgânica ou mineral.

O tratamento com plasma, que consiste no quarto estado da matéria, tem sido amplamente utilizado nas indústrias aeroespacial, ambiental, energética e eletrônica ${ }^{25}$, visto que o mesmo libera radicais livres que aumentam a energia livre e a molhabilidade de superfícies ${ }^{26}$. Na área médica, o tratamento com plasma atmosférico não-térmico (PANT) pode ser utilizado com segurança, devido ao seu efeito antioxidante, anti-inflamatório e bactericida, sem que haja aumento na temperatura ${ }^{27,28}$. Na Odontologia, o PANT tem sido bastante estudado tanto com relação a suas propriedades terapêuticas ${ }^{29-32}$, quanto com relação a sua capacidade de melhorar a adesão ${ }^{33-37}$. Sabe-se que o PANT aumenta a penetração de adesivos na dentina desmineralizada ${ }^{38,39}$, e é capaz de melhorar a adesão a esse tecido dental ${ }^{35-37}$, bem como à materiais protéticos a base de zircônia ${ }^{33,34}$. Recentemente, $\mathrm{o}$ PANT foi sugerido em técnicas de reparo de restaurações diretas de resina composta ${ }^{40}$ e também poderia ser aplicado nos novos materiais CAD/CAM, quando houver a necessidade de reparo.

A umidade da cavidade oral desempenha um papel importante na degradação da adesão, sendo assim um fator relevante a ser estudado, já que pode influenciar na longevidade do reparo às $\mathrm{CMRs}^{41}$. A degradação hidrolítica da matriz monomérica das CMRs, bem como da resina composta utilizada no reparo, pode induzir uma maior falha da adesão à CMRs a longo prazo ${ }^{8-10}$. Apesar de diversos trabalhos terem avaliado o reparo com resina composta à $\mathrm{CMRs}^{14-24}$, a avaliação do efeito do envelhecimento sobre essa adesão ainda precisa ser melhor explorado ${ }^{15,17,19,20}$.

Portanto, o objetivo deste trabalho foi avaliar o efeito do tratamento com PANT, sozinho ou associado ao jateamento com óxido de alumínio ou aplicação de adesivo, e do tempo de avaliação após armazenamento em água na RUC do reparo de CMRs com resina composta. As hipóteses nulas deste trabalho foram que: (1) o tratamento com PANT, associado ou não ao jateamento ou adesivo, não aumentaria a RUC do reparo; (2) a imersão em água por 1 ano não diminuiria a RUC do reparo às CMRs.

\section{MATERIAL E MÉTODOS}

As marcas comerciais, fabricantes, composições e números de lote dos materiais utilizados neste trabalho estão listados na Tabela 1. Três tipos diferentes de CMRs, foram testadas: Vita Enamic (VITA Zahnfabrik, Bad Säckingen, Alemanha), Cerasmart (GC Corp., Tóquio, Japão) e Lava Ultimate (3M Oral Care, St. Paul, MN, EUA).

Os blocos de CMRs foram seccionadas em placas (14 x $7 \times 1$ $\mathrm{mm}$ ), utilizando um disco diamantado montado em uma máquina de corte de precisão (Isomet 1000 Precision Saw; Buehler Co, Lake Bluff, IL, EUA). Buscando padronizar a superfície das placas, as mesmas foram polidas com lixas abrasivas de óxido de alumínio (T216 Sandpaper, Norton, Guarulhos, SP, Brasil) de granulação 600, utilizando uma politriz (Automet 500, Buehler Co, Lake Bluff, IL, EUA).

As amostras foram fixadas com adesivo termoplástico (Bmfctrans, Bumafer, Palmeira, PR, Brasil) a uma máquina de envelhecimento artificial acelerado (EQ-UV, Equilam, Diadema, SP, Brasil). A programação de envelhecimento consistiu em 4 horas de envelhecimento com luz UV-B, seguida de 4 horas de condensação de umidade, ambos a $50^{\circ} \mathrm{C}$, durante um total de 300 horas, que correspondem a 1 ano de envelhecimento clínico ${ }^{42}$.

Visando delimitar as áreas de adesão nas placas, duas fitas adesivas, cada uma com um orifício de 1,5 mm de diâmetro, foram fixadas na superfície de cada placa. Estas foram divididas em quatro grupos, de acordo com o tratamento de superfície realizado:

1. (CT) - Controle: jateamento com óxido de alumínio $(50 \mu \mathrm{m})$ com uma unidade de jateamento (Microetcher II; Danville Materials Inc., San Ramon, CA, EUA) durante 10 s, com uma pressão de $60 \mathrm{psi}$, a uma distância de $10 \mathrm{~mm}$. As amostras foram então lavadas e submetidas a banho em ultrassom (USC 1400; Unique, Indaiatuba, SP, Brasil) com água destilada por 5 minutos. Posteriormente, as amostras foram secas e uma camada de agente de união silano (RelyX Ceramic Primer; 3M Oral Care, St. Paul, MN, EUA) foi aplicada durante $10 \mathrm{~s}$. Após secagem com jato de ar por $10 \mathrm{~s}$, a uma distância de $10 \mathrm{~mm}$, uma camada de adesivo hidrófobo (Adhesive - Scotchbond Multipurpose; 3M Oral Care, St. Paul, MN, EUA) foi aplicada, seguida de jato de ar por $10 \mathrm{~s}$, e fotoativação por $10 \mathrm{~s}$ com um aparelho fotoativador (1036.1 mW/cm² de emitância, Bluephase Style; Ivoclar Vivadent, Schaan, Liechtenstein);

Tabela 1 - Marca comercial, fabricante, composição e lote dos materiais utilizados.

\begin{tabular}{lll}
\hline Material & Fabricante & Composição \\
\hline Enamic & Vita Zahnfabrik & $\begin{array}{l}14 \% \text { (peso) orgânico: TEGDMA e UDMA } \\
86 \% \text { (peso) inorgânico: cerâmica feldspática e alumina }\end{array}$ \\
\hline Cerasmart & GC Corp. & $\begin{array}{l}29 \% \text { (peso) orgânico: Bis-MEPP, UDMA, dimetacrilato } \\
71 \% \text { (peso) inorgânico: sílica, nanopartículas de bário }\end{array}$ \\
\hline Lava Ultimate & 3M Oral Care & $\begin{array}{l}20 \% \text { (peso) orgânico: Bis-GMA, UDMA, Bis-EMA, TEGDMA } \\
80 \% \text { (peso) inorgânico: silica, nanopartículas de zirconia }\end{array}$ \\
\hline RelyX Ceramic Primer & 3M Oral Care & Etanol, água, metacriloxipropiltrimetoxi-silano \\
\hline Scotchbond Multipurpose & 3M Oral Care & Adhesive (3): Bis-GMA, HEMA, trifenilantimônio, aminas terciárias \\
\hline & & $\begin{array}{l}\text { Bis-GMA modificado por uretano, Bis-EMA, UDMA, TEGDMA, sílica amorfa, vidro de bário-boro- } \\
\text {-flúor-alumino silicato, vidro de bário-boro-alumino silicato, hidrófobo coloidal, benzofenona III, } \\
\text { concentrado flublau, EDAB, CQ, BHT, óxido de ferro amarelo, óxido de ferro vermelho, óxido de ferro 2468901 } \\
\text { preto e dióxido de titânio }\end{array}$ \\
\hline TEGDMA, trietileno glicol dimetacrilato; UDMA, uretano dimetacrilato; Bis-MEPP, bis[2-(metacriloiloxi)etil] fosfato; Bis-GMA, bisfenol A diglicidil éter dimetacrilato; Bis-EMA, bisfe- \\
nol A glicol dimetacrilato etoxilado; HEMA, hidroxietil metacrilato; EDAB, amina dimetilaminoetil benzoato; CQ, canforquinona; BHT, hidroxitolueno butilado.
\end{tabular}


2. (PL): Aplicação de PANT de gás argônio (KinPen 09, INP; Greifswald, Alemanha) com uma taxa de $5 \mathrm{~L} / \mathrm{min}$, a uma distância de $10 \mathrm{~mm}$, perpendicular à superfície da amostra, durante 30s. As fitas adesivas foram aplicadas à superfície após aplicação do PANT (Figura 1);

3. (PA): Aplicação de PANT, seguido de adesivo hidrófobo, conforme descrito anteriormente;

4. (JP): Jateamento com óxido de alumínio, seguido da aplicação PANT conforme descrito previamente.

Após os tratamentos de superfície das placas, dois moldes de silicone de adição (Virtual; Ivoclar Vivadent, Schaan, Liechtenstein), cada um com um orifício de 1,5 mm de diâmetro e uma altura de $1,5 \mathrm{~mm}$, foram posicionados sobre a superfície tratada das amostras com seus orifícios concêntricos aos orifícios das fitas adesivas. Os orifícios dos moldes de silicone foram então completamente preenchidos com uma resina composta (Spectra Smart; Dentsply Sirona; Konstanz, Alemanha), a qual foi fotoativada durante 20s com um aparelho fotoativador $(1036,1 \mathrm{~mW} /$ $\mathrm{cm}^{2}$ de emitância, Bluephase Style; Ivoclar Vivadent, Schaan, Liechtenstein), obtendo assim dois cilindros de resina composta polimerizados em cada placa. Os moldes de silicone e fitas adesivas foram cuidadosamente removidos e as amostras armazenadas em água destilada a $37^{\circ} \mathrm{C}$. Para cada placa um cilindro de resina composta foi submetido ao teste de RUC após $24 \mathrm{~h}$ armazenamento, e o outro após 1 ano $(\mathrm{n}=10)$.

Previamente ao teste de RUC, as amostras foram secas e fixadas com cola de cianoacrilato (Super Bonder; Loctite, São Paulo, SP, Brasil) a um dispositivo de teste, o qual foi acoplado a uma máquina de ensaio universal (EZ-Test; Shimadzu, Quioto, Japão). A carga foi aplicada na base do cilindro de resina composta com um fio ortodôntico ( $2 \mathrm{~mm}$ de diâmetro), em velocidade de 1,0 mm/min até a falha. Os valores de RUC foram calculados dividindo a carga máxima na falha (Newton) pela área de adesão $\left(\mathrm{mm}^{2}\right)$ e expressos em megapascal (MPa). Os dados de RUC apresentaram normalidade e homocedacidade e foram analisados por ANOVA três fatores (material, tratamento de superfície

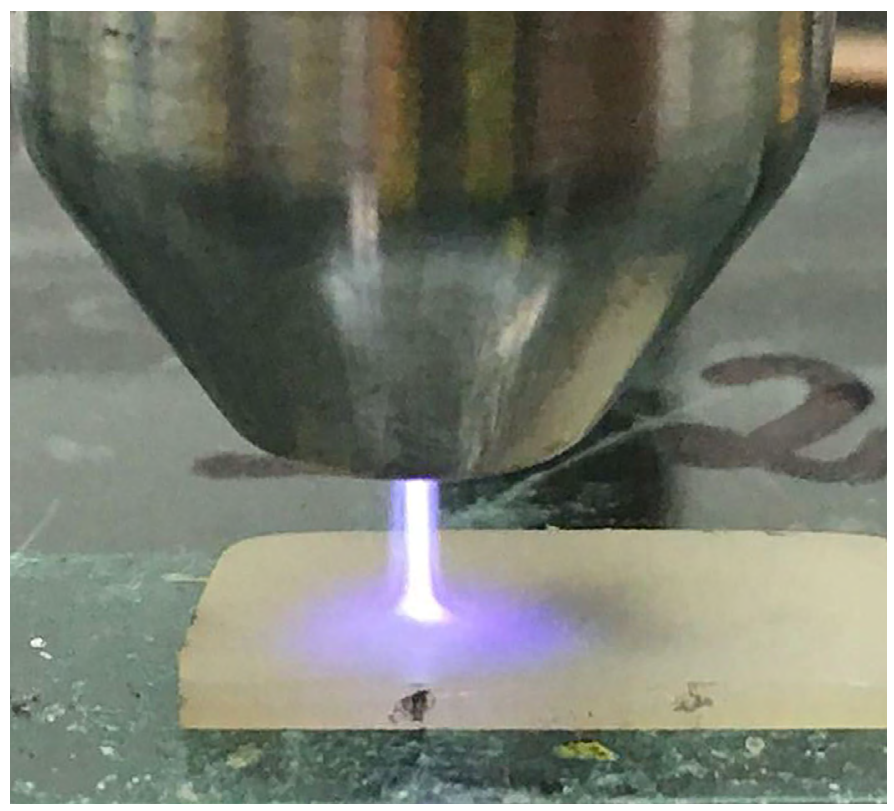

Figura 1 - Aplicação do PANT na superfície de uma CMR. e tempo de armazenamento em água), seguido de teste de Tukey ( $\alpha=5 \%$ ).

Após o teste de RUC a área de união das amostras foi analisada em microscópio digital (KH 8700; Hirox, Tóquio, Japão) e micrografias foram obtidas em aumento de 100x, para classificá-las quanto aos seguintes padrões de fratura: 1 - falha adesiva (ADE); 2 - falha coesiva em CMR (COC); 3 - falha mista entre adesiva e coesiva em resina (MAR); 4 - falha mista entre adesiva e coesiva em CMR (MAC); 5 - falha mista entre adesiva, e coesivas em resina e CMR (MARC).

Adicionalmente, amostras das CMRs nas dimensões de $14 \times 2$ x $2 \mathrm{~mm}(\mathrm{n}=2)$ foram preparadas e fraturadas em máquina de ensaio universal (EZ-Test; Shimadzu, Quioto, Japão) para avaliação estrutural do material. As superfícies fraturadas foram cobertas com ouro (SCD 050; Bal-tec, Balzers, Liechtenstein) para posterior análise em microscópio eletrônico de varredura (MEV) (JSM IT 300; JEOL, Tóquio, Japão) e registro de imagens com 5.000x de aumento.

Para a análise das partículas de carga inorgânicas da resina composta reparadora, uma porção não-polimerizada da mesma (1 mg) foi submersa em $6 \mathrm{~mL}$ de acetona 99.5\% (Merck KGA, Darmstadt, Alemanha) e submetida à centrifugação (Excelsa, modelo 206, FANEM, São Paulo, SP, Brasil) por 5 minutos. Este processo foi repetido até que nenhum sobrenadante fosse identificado após a centrifugação. $\mathrm{O}$ mesmo protocolo de submersão e centrifugação foi realizado com clorofórmio 99.8\% (Merck KGA, Darmstadt, Alemanha) e posteriormente com etanol absoluto (Merck KGA, Darmstadt, Alemanha). As partículas foram então armazenadas em etanol absoluto por $24 \mathrm{~h}$, em uma estufa (FANEM, São Paulo, SP, Brasil) a $37^{\circ} \mathrm{C}^{43}$. Finalmente, as partículas foram aplicadas em uma fita de carbono, aderida a um stub metálico, e submetidas à cobertura com ouro e análise em MEV, conforme descrito anteriormente. Micrografias com aumento de 5.000x foram obtidas das partículas de carga da resina composta reparadora.

\section{RESULTADOS}

Os resultados obtidos para a RUC, bem como os valores de redução percentual da média de cada grupo de $24 \mathrm{~h}$ para 1 ano, se encontram na Tabela 2. A análise estatística revelou que a RUC foi significativamente influenciada pelos três fatores estudados: "material" ( $p=0,0293)$, "tratamento de superfície" $(p<0,0001)$ e "tempo de armazenamento" ( $p=0,0041)$. A interação entre os fatores "material" e "tratamento de superfície", e "material" e "tempo de armazenamento" também teve influencia significativa para os valores de RUC ( $p<0,0001)$. Entretanto, a interação entre os fatores "tratamento de superfície" e "tempo de armazenamento" não foi significante ( $p=0.6992)$. Por fim, a interação dos três fatores estudados teve influência significativa nos valores de RUC ( $\mathrm{p}=0,0059)$.

No tempo de $24 \mathrm{~h}$, para os tratamentos CT e PL, Enamic obteve valores de RUC superiores à Cerasmart e Lava Ultimate $(\mathrm{p}<$ $0,05)$, os quais não diferiram entre si $(\mathrm{p}>0,05)$. Já para os tratamentos PA e JP, não houve diferença na RUC entre os materiais $(\mathrm{p}>0,05)$. Entretanto, após 1 ano de imersão em água para o tratamento CT, Enamic não diferiu de Cerasmart ( $\mathrm{p}>0,05)$, porém foi superior a Lava Ultimate $(p<0,05)$, enquanto este não diferiu de Cerasmart $(p>0,05)$. Para os demais tratamentos (PA, JP e PL) 
não houve diferença na RUC para todos os materiais ( $p>0,05)$.

Considerando os tratamentos de superfície para as amostras testadas em $24 \mathrm{~h}$, o grupo CT não diferiu do PA para Cerasmart e Lava Ultimate $(p>0,05)$ e foi superior aos tratamentos JP e PL $(p<0,05)$, enquanto para Enamic, o tratamento CT obteve valores superiores a todos tratamentos $(\mathrm{p}<0,05)$. O tratamento JP foi inferior a PA e CT para todos materiais $(\mathrm{p}<0,05)$. Já o tratamento $\mathrm{PL}$, foi inferior aos demais (CT, PA e JP) para os materiais Cerasmart e Lava Ultimate $(p<0,05)$, enquanto para Enamic, PL foi inferior ao CT $(p<0,05)$, porém não diferiu de PA e JP $(p>0,05)$.

Avaliando-se os tratamentos de superfície para as amostras testadas com 1 ano de armazenamento, o tratamento CT foi superior aos demais para Enamic e Cerasmart $(p<0,05)$, enquanto para a Lava Ultimate, CT foi superior ao PA e PL $(p<0,05)$, porém não diferiu de JP $(p>0,05)$. Para Enamic e Cerasmart, os tratamentos PA e JP não diferiram entre si ( $p>0,05)$, e foram superiores ao PL $(p<0,05)$. Para a Lava Ultimate, o tratamento JP não diferiu de PA $(p>0,05)$, porém foi superior a PL $(p<0,05)$, o qual não diferiu de PA ( $p>0,05)$.

Os padrões de fratura identificados (Figura 2), por ordem de prevalência, foram: falha adesiva (ADE - 94,2\%), falha mista entre adesiva e coesiva em resina (MAR - 3,3\%), falha mista entre adesiva e coesiva em CMR (MAC - 1,3\%), falha coesiva em CMR (COC - 0,8\%) e falha mista entre adesiva e coesivas em resina e CMR (MARC - 0,4\%) (Figura 3).

As falhas MAC e MARC foram identificadas somente no tratamento CT para Enamic em 24h. A falha COC foi identificada também no tratamento $\mathrm{CT}$ do Enamic, e no tratamento PA do Lava Ultimate, ambos em $24 \mathrm{~h}$. Por fim, a falha MAR foi identificada para o Enamic com tratamento CT e PA em 24 h e tratamento CT com 1 ano, bem como também foi identificada para o Cerasmart com tratamento CT e JP, e para o Lava Ultimate com tratamento CT e PL, todos em 24h.

As micrografias evidenciaram a rede polimérica infiltrada na matriz inorgânica cerâmica do Enamic (Figura 4A). Pode-se observar a presença de partículas de carga esféricas para Cerasmart (Figura 4B) e Lava Ultimate (Figura 4C), sendo as partículas do Cerasmart visivelmente menores que as do Lava Ultimate. Observando a micrografia das partículas de carga da resina composta utilizada como reparo das CMRs (Figura 4D), pode-se notar que as mesmas possuem formato irregular e tamanho variável de 0,5 a $4 \mu \mathrm{m}$.

\section{DISCUSSÃO}

Apesar da comprovada melhoria na adesão promovida pelo PANT quando aplicado à dentina ${ }^{35-37}$ e à zircônia ${ }^{33,34}$, este mesmo efeito não foi observado neste trabalho que simulou reparos nas CMRs tratadas com PANT. Portanto, a primeira hipótese nula deste trabalho foi aceita, visto que o tratamento com PANT, mesmo associado ao jateamento ou a aplicação de adesivo hidrófobo, não aumentou a RUC do reparo das CMRs com resina composta. Assim, o tratamento com jateamento de óxido de alumínio, silano e adesivo hidrófobo deve ser indicado para o reparo de CMRs. Por se tratarem de estruturas híbridas, com uma matriz orgânica e uma inorgânica, a adesão a estes materiais pode ser desafiadora. Por um lado, a matriz orgânica possui uma polimerização industrial com pressão e temperatura controladas, que provavelmente resulta em poucos remanescentes monoméricos

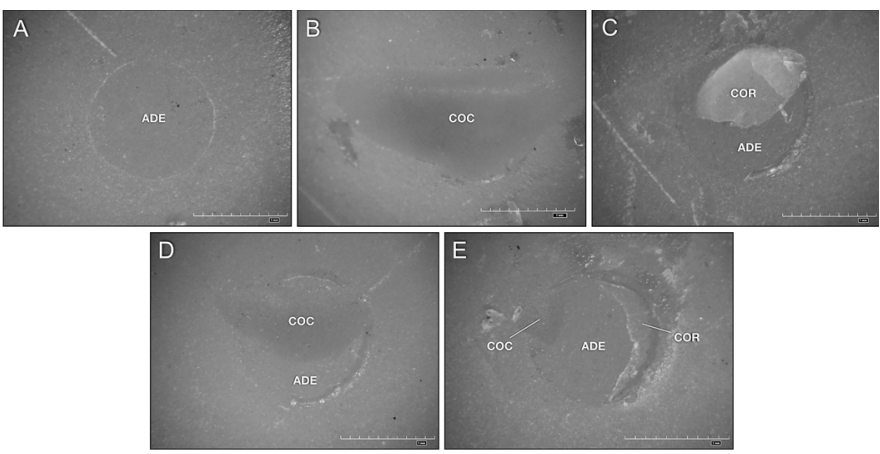

Figura 2 - Padrões de fratura identificados nas amostras após ensaio de cisalhamento: A, falha adesiva (ADE); B, falha coesiva em CMR (COC); C, falha mista entre adesiva e coesiva em resina; $\mathrm{D}$, falha mista entre adesiva e coesiva em CMR (COC); $\mathrm{E}$, falha mista entre adesiva, e coesivas em resina e CMR.

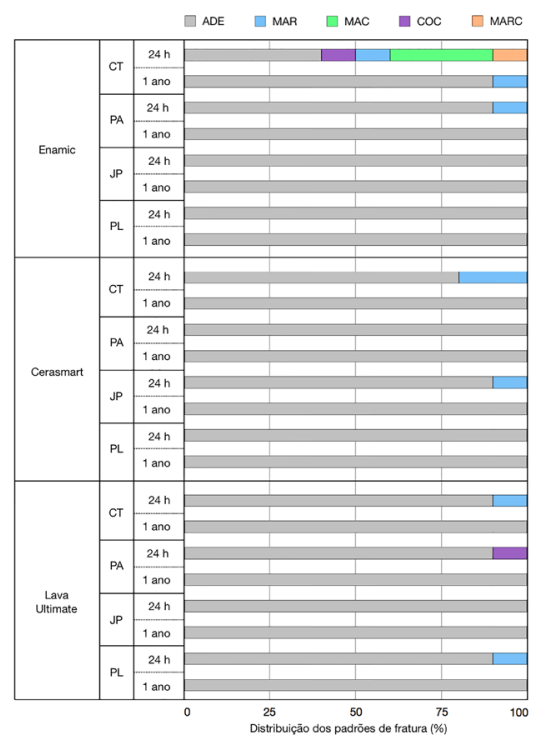

Figura 3 - Distribuição percentual dos padrões de fratura para cada grupo experimental: controle (CT), PANT + adesivo (PA), jateamento + PANT (JP) e PANT (PL). $\mathrm{ADE}$, falha adesiva. MAR, falha mista entre adesiva e coesiva em resina. MAC, falha mista entre adesiva e coesiva em CMR. COC, falha coesiva em CMR. MARC, falha mista entre adesiva e coesivas em resina e CMR.
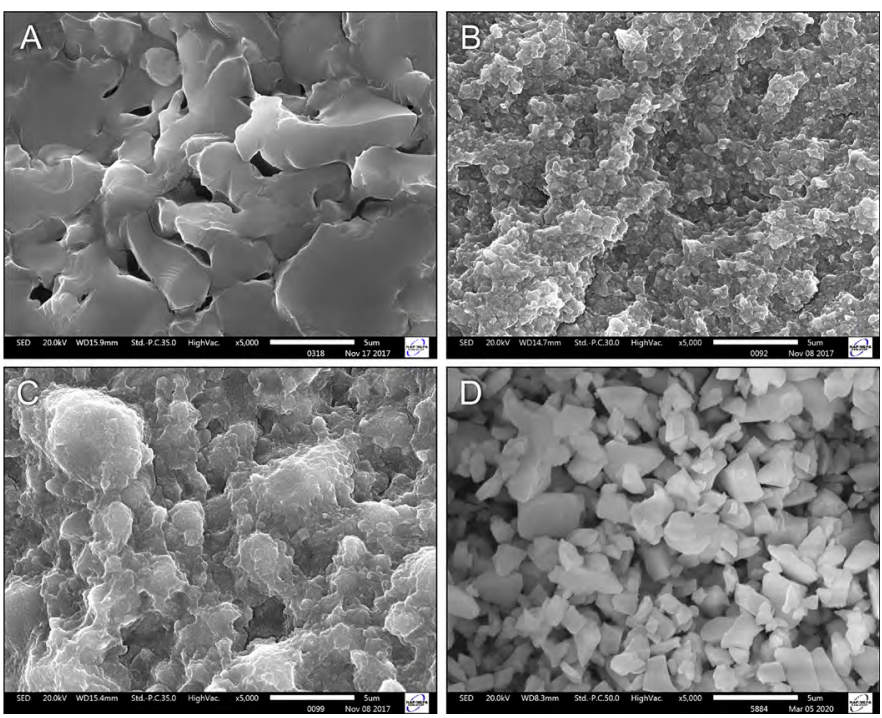

Figura 4 - Micrografias das CMRs (A, Enamic; B, Cerasmart; C, Lava Ultimate) e das partículas de carga da resina composta reparadora Spectra Smart em magnificação de $5.000 x$ (D). 
não reagidos. Esses monômeros, mais abundantes em resinas compostas com polimerização ativada por luz, funcionam como sítios de ligação para os monômeros presentes no adesivo ou na resina composta reparadora. Por outro lado, a porção inorgânica está recoberta pela matriz orgânica, o que pode diminuir os sítios de ligação do agente de união silano.

Neste estudo o PANT foi utilizado de forma isolada (PL), ou associado ao jateamento com óxido de alumínio (JP) ou adesivo hidrófobo (PA). Apesar da aplicação do adesivo no tratamento PA ter obtido bons resultados após $24 \mathrm{~h}$, principalmente para Cerasmart e Lava Ultimate que não diferiram do CT, após 1 ano esses valores reduziram drasticamente $(48,8 \%$ para Cerasmart, $51,0 \%$ para Enamic e $61,4 \%$ para Lava Ultimate). Mesmo assim, os grupos PA (exceto para Lava Ultimate) e JP obtiveram resultados superiores de RUC comparados ao grupo PL após 1 ano, evidenciando que o PANT deve ser utilizado associado a outros tratamentos de superfície nas técnicas de reparo de CMRs.

Além disso, não houve aplicação do agente de união silano em nenhum dos grupos tratados com PANT, o que pode ajudar a explicar o pior desempenho destes grupos comparados ao CT. O silano consiste em uma molécula bifuncional capaz de promover união entre a sílica e os monômeros metacrilatos ${ }^{44}$ applications of silane coupling agents and related surface pretreatment methods in contemporary dentistry. Methods: Silane coupling agents are adhesion promoters to chemically unify dissimilar materials used in dentistry. Silanes are very effective in adhesion promotion between resin composites and silica-based or silica-coated indirect restorative materials. It is generally accepted that for non-silica-based restorations, surface pretreatment is a mandatory preliminary step to increase the silica content and then, with help of silane, improve resin bonding. This review discusses the silane-based adhesion chemistry, silane applications in dentistry, surface pretreatment methods, and presents the recent development of silane coupling agents. Results: A silane coupling agent is considered a reliable, good adhesion promoter to silica-based (or silica-coated. Alguns estudos comprovaram que o uso deste agente de união aumentou a adesão de reparos de resina composta às $\mathrm{CMRs}^{16,20,22}$. O uso de silano após aplicação do PANT poderia promover resultados diferentes dos obtidos neste estudo, o que deve ser objeto de estudos futuros.

As CMRs também estão sujeitas à degradação hidrolítica no ambiente oral, que pode diminuir a adesão em situações de reparo com resina composta. Essa degradação se dá tanto no adesivo e resina composta utilizados no reparo, quanto na CMR, devido à hidrólise do grupamento éster dos monômeros metacrilatos, e da ligação entre silano e o conteúdo inorgânico ${ }^{41}$. A degradação pode explicar a diminuição da RUC observada na maioria dos grupos testados neste estudo após 1 ano de imersão em água. Assim, a segunda hipótese nula foi rejeitada, visto que o armazenamento em água reduziu a RUC do reparo em resina composta às CMRs para vários tratamentos de superfície testados.

Não obstante, as CMRs possuíram um comportamento bastante diferente frente à degradação pela imersão em água. Para o tratamento $\mathrm{CT}$, por exemplo, a redução do valor da média de RUC de 24 h para 1 ano, foi de 53,0\% para Enamic, 39,7\% para Lava Ultimate e somente 2,8\% para Cerasmart, o qual não apresentou diferença estatística entre $24 \mathrm{~h}$ e 1 ano. Este resultado indica que a adesão à Cerasmart obteve uma estabilidade
Tabela 2 - Média (DP) da RUC, em MPa, do reparo das CMRs com resina composta em função do tratamento de superfície e tempo de armazenamento em água, e a diminuição percentual (\%) da média da RUC de 24 h para 1 ano.

\begin{tabular}{lllll}
\multirow{2}{*}{ Tempo } & \multirow{4}{*}{$\begin{array}{l}\text { Tratamento } \\
\text { de Superfície }\end{array}$} & & & \\
\end{tabular}

Médias seguidas de letras distintas (maiúsculas na horizontal, comparando CMRs para o mesmo tratamento e tempo de avaliação; e minúsculas na vertical, comparando tratamentos dentro da mesma CMR e tempo) diferem entre si $(p \leq 0,05)$. * Difere de $24 \mathrm{~h}$ nas mesmas condições de tratamento e para o mesmo CMR ( $p \leq$ 0,05). Abreviações: CMR - cerâmica de matriz resinosa, CT - controle, PA - plasma + adesivo, JP - jateamento + plasma, PL - plasma.

consideravelmente superior aos demais materiais. Apesar do resultado após 1 ano da Enamic ser numericamente superior à Cerasmart, não houve diferença estatística entre os valores de RUC, e o fato de Enamic ter obtido uma redução tão expressiva da RUC com 1 ano (53,0\%), pode levar à hipótese que a adesão a esse material é menos duradoura, seguindo os protocolos de tratamento de superfície deste estudo. Entretanto, um trabalho de Silva et al. ${ }^{17}$ (2018), detectou que a adesão de um reparo à Enamic não alterou após 6 meses de armazenamento em água quando o mesmo foi submetido a tratamento de superfície com jateamento com óxido de alumínio, silano e adesivo. Devido à escassez de artigos que avaliaram a adesão do reparo ao material Enamic após algum tipo de envelhecimento, a estabilidade da adesão promovida a esse material carece de mais estudos.

A grande porcentagem de conteúdo inorgânico do Enamic ( $86 \%$ em peso) faz com que o fabricante indique o uso de ácido fluorídrico (HF) $5 \%$ visando aumentar a rugosidade superficial do material. De fato, a adesão de cimentos resinosos na superfície do Enamic é superior quando tratada com HF 5\%, comparado ao tratamento de jateamento com óxido de alumínio ${ }^{8}$ marginal adaptation, and bond strengths of inlays made of computer-aided design/computer-aided manufacturing feldspathic ceramic and polymer-infiltrated ceramic. Twenty molars were randomly selected and prepared to receive inlays that were milled from both materials. Before cementation, internal fit was achieved using the replica technique by molding the internal surface with addition silicone and measuring the cement thicknesses of the pulpal and axial walls. Marginal adaptation was measured on the occlusal and proximal margins of the replica. The inlays were then cemented using resin cement (Panavia F2.0. Isto poderia explicar a pior estabilidade da RUC do Enamic comparada aos demais materiais. Entretanto, alguns estudos encontraram que não houve diferença na resistência de união do reparo ao Enamic quando tratada com HF ou jateada após 24 h de imersão em água ${ }^{16,17,20,22}$. 
Além disso, a aplicação HF obteve desempenho inferior ao jateamento com óxido de alumínio e silicatização, após 6 meses de armazenamento em água ${ }^{17}$. Portanto, estudos avaliando a adesão ao longo do tempo do reparo no material Enamic são importantes para definir se o HF deve ou não ser empregado.

De forma oposta, Cerasmart é a CMR com maior quantidade de matriz orgânica ( $29 \%$ em peso), e a RUC do reparo não mostrou alteração após 1 ano de armazenamento em água. A grande quantidade de matriz orgânica, aumenta a possibilidade de grupamentos metacrilato pendentes disponíveis para união com o adesivo e resina composta do reparo, podendo assim aumentar a estabilidade da união mesmo após 1 ano de imersão em água. A literatura ainda carece de informações quanto ao melhor protocolo de tratamento de superfície para o reparo do material Cerasmart ${ }^{21}$.

De forma geral, a maior parte dos estudos sobre reparo à CMRs avaliaram a adesão somente após 24 ou $48 \mathrm{~h}$ de imersão em água ${ }^{14,16,18,21-24}$, ou somente após termociclagem ${ }^{15,19,20}$. Apenas um estudo comparou a adesão imediata com a adesão após a termociclagem ${ }^{17}$. A avaliação de forma isolada, imediata ou após envelhecimento, impede a percepção da degradação da adesão promovida a longo prazo, podendo induzir a interpretações e conclusões equivocadas com relação à longevidade do tratamento de superfície realizado. Portanto, nosso estudo traz uma importante contribuição para a Literatura Odontológica quanto à estabilidade e longevidade do reparo de CMRs com resina composta restauradora direta.

No geral, o padrão de fratura mais prevalente foi a falha adesiva (94,2\%). Entretanto, o tratamento CT na Enamic em $24 \mathrm{~h}$ chamou atenção por possuir $60 \%$ de falhas coesivas ou mistas. Esse resultado está em concordância ao resultado de RUC, o qual foi bastante superior às demais CMRs em $24 \mathrm{~h}$. Sabe-se que o ensaio de cisalhamento pode induzir uma maior taxa de falhas coesivas, ou mistas, devido à concentração de tensões na base do cilindro resinoso ${ }^{45}$. Entretanto, percebe-se que grupos com maior RUC tendem a gerar maior quantidade de falhas não adesivas, fato esse observado neste trabalho, visto que, com exceção ao tratamento CT na Enamic, essas falhas se encontraram sempre nos resultados em $24 \mathrm{~h}$, nos quais a RUC também tendeu a ser superior.

Outros tratamentos de superfície têm sido estudados para melhorar a adesão do reparo às CMRs, como a aplicação de adesivos universais ${ }^{15,17,21-23}$ e a silicatização ${ }^{14-17,20,21,23,24}$. O uso do PANT na técnica de reparo de materiais CAD/CAM, associado a esses tratamentos também deve ser avaliado em futuras investigações. $\mathrm{O}$ armazenamento por um ano em água proporcionou a avaliação do efeito da degradação hidrolítica na RUC do reparo das CMRs envelhecidas artificialmente com resina composta. Além da água, outros fatores também podem contribuir para a degradação in vivo da área de união do reparo, como alterações de temperatura e $\mathrm{pH}$, fadiga oclusal e presença de microbiota bucal. Assim, outros estudos devem ser conduzidos afim de avaliar o efeito desses fatores individuais na adesão do reparo com resina composta às CMRs.

\section{CONCLUSÃO}

Com base nos resultados deste estudo in vitro, pode-se concluir que: a. O tratamento com PANT, associado ou não com jateamento ou adesivo, não promoveu aumento da RUC do reparo com resina composta às CMRs.

b. O tratamento com jateamento, aplicação de silano e adesivo hidrófobo (controle) produziu os maiores valores de RUC, sendo, portanto, o método sugerido para o reparo das CMRs, principalmente avaliando-se a longevidade.

\section{AGRADECIMENTOS}

Gostaríamos de agradecer ao Dr. Elliot Watanabe Kitajima e ao técnico Renato Barbosa Salaroli, Laboratório de Microscopia Eletrônica aplicada à Pesquisa Agropecuária (NAP/MEPA) da Escola Superior de Agricultura "Luiz de Queiroz" da Universidade de São Paulo, Piracicaba, SP, Brasil (ESALQ/USP) pela assistência com as análises microscópicas deste estudo e à Profa. Dra. Gláucia Maria Bovi Ambrosano da Faculdade de Odontologia de Piracicaba da Universidade de Campinas, Piracicaba, SP, Brasil (FOP-UNICAMP) pelo auxílio com a análise estatística.

\section{REFERÊNCIAS}

01. Belli R, Wendler M, Ligny D, Cicconi MR, Petschelt A, Peterlik H, et al. Chairside CAD/CAM materials. Part 1: Measurement of elastic constants and microstructural characterization. Dent Mater. 2017; 33(1):84-98.

02. Yoshihara K, Nagaoka N, Maruo Y, Nishigawa G, Irie M, Yoshida Y, et al. Sandblasting may damage the surface of composite CAD-CAM blocks. Dent Mater. 2017; 33(3):e124-35.

03. Chavali R, Nejat AH, Lawson NC. Machinability of CAD-CAM materials. J Prosthet Dent. 2017; 118(2): 194-9.

04. Awada A, Nathanson D. Mechanical properties of resin-ceramic CAD/CAM restorative materials. J Prosthet Dent. 2015; 114(4):587-93.

05. Lebon N, Tapie L, Vennat E, Mawussi B. Influence of CAD/CAM tool and material on tool wear and roughness of dental prostheses after milling. J Prosthet Dent. 2015; 114(2):236-47.

06. Gracis S, Thompson V, Ferencz J, Silva N, Bonfante E. A New Classification System for All-Ceramic and Ceramic-like Restorative Materials. Int J Prosthodont. 2016; 28(3):227-35.

07. Ab-Ghani Z, Jaafar W, Foo S, Ariffin Z, Mohamad D. Shear bond strength of computer-aided design and computer-aided manufacturing feldspathic and nano resin ceramics blocks cemented with three different generations of resin cement. J Conserv Dent. 2015; 18(5):355-9.

08. Campos F, Almeida C, Rippe M, de Melo R, Valandro LF, Bottino M. Resin Bonding to a Hybrid Ceramic: Effects of Surface Treatments and Aging. Oper Dent. 2016; 41(2):171-8.

09. Cekic-Nagas I, Ergun G, Egilmez F, Vallittu PK, Lassila LVJ. Microshear bond strength of different resin cements to ceramic/glasspolymer CAD-CAM block materials. J Prosthodont Res. 2016; 60(4):265-73.

10. Elsaka SE. Bond strength of novel CAD/CAM restorative materials to self-adhesive resin cement: the effect of surface treatments. J Adhes Dent. 2014; 16(6):531-40.

11. Kassotakis EM, Stavridakis M, Bortolotto T, Ardu S, Krejci I. Evaluation of the Effect of Different Surface Treatments on Luting CAD/CAM Composite Resin Overlay Workpieces. J Adhes Dent. 2015; 17(6):521-8.

12. Park JH, Choi YS. Microtensile bond strength and micromorphologic analysis of surface-treated resin nanoceramics. J Adv Prosthodont. 2016; 8(4): 275. 
13. Poggio C, Pigozzo M, Ceci M, Scribante A, Beltrami R, Chiesa $\mathrm{M}$. Influence of different luting protocols on shear bond strength of computer aided design/computer aided manufacturing resin nanoceramic material to dentin. Dent Res J. 2016; 13(2):91-7.

14. Zaghloul H, Elkassas DW, Haridy MF. Effect of incorporation of silane in the bonding agent on the repair potential of machinable esthetic blocks. Eur J Dent. 2014; 8(1):44-52.

15. Stawarczyk B, Krawczuk A, Ilie N. Tensile bond strength of resin composite repair in vitro using different surface preparation conditionings to an aged CAD/CAM resin nanoceramic. Clin Oral Investig. 2015; 19(2):299-308.

16. Elsaka SE. Repair bond strength of resin composite to a novel CAD/ CAM hybrid ceramic using different repair systems. Dent Mater J. 2015; 34(2):161-7.

17. Silva PNF, Martinelli-Lobo CM, Bottino MA, Melo RM, Valandro LF. Bond strength between a polymer-infiltrated ceramic network and a composite for repair: effect of several ceramic surface treatments. Braz Oral Res. 2018; 32:e28.

18. Shinohara A, Taira Y, Sakihara M, Sawase T. Effects of three silane primers and five adhesive agents on the bond strength of composite material for a computer-aided design and manufacturing system. J Appl Oral Sci. 2018; 26:1-7.

19. Üstün Ö, Büyükhatipoğlu IK, Seçilmiş A. Shear Bond Strength of Repair Systems to New CAD/CAM Restorative Materials. J Prosthodont. 2018; 27(8):748-54.

20. Tatar N, Ural C. Repair Success of Two Innovative Hybrid Materials as a Function of Different Surface Treatments. Int J Prosthodont. 2018; 31(3):267-70.

21. Demirel G, Baltacioğlu İH. Influence of different universal adhesives on the repair performance of hybrid CAD-CAM materials. Restor Dent Endod. 2019; 44(3):1-9.

22. Bello YD, Di Domenico MB, Magro LD, Lise MW, Corazza PH. Bond strength between composite repair and polymer-infiltrated ceramicnetwork material: Effect of different surface treatments. J Esthet Restor Dent. 2019; 31(3):275-9.

23. Arpa C, Ceballos L, Fuentes MV, Perdigão J. Repair bond strength and nanoleakage of artificially aged CAD-CAM composite resin. J Prosthet Dent. 2019; 121(3):523-30.

24. Wiegand A, Stucki L, Hoffmann R, Attin T, Stawarczyk B. Repairability of CAD/CAM high-density PMMA and composite-based polymers. Clin Oral Investig. 2015; 19(8):2007-13.

25. Kim JH, Lee MA, Han GJ, Cho BH. Plasma in dentistry: A review of basic concepts and applications in dentistry. Acta Odontol Scand. 2014; 72(1):1-12

26. Tendero C, Tixier C, Tristant P, Desmaison J, Leprince P. Atmospheric pressure plasmas: A review. Spectrochim Acta Part B. At Spectrosc. 2006; 61(1):2-30

27. Fridman G, Friedman G, Gutsol A, Shekhter AB, Vasilets VN, Fridman A. Applied plasma medicine. Plasma Process Polym. 2008; 5(6):503-33.

28. Hoffmann C, Berganza C, Zhang J. Cold Atmospheric Plasma: Methods of production and application in dentistry and oncology. Med Gas Res. 2013; 3(1):1-15.
29. Ermolaeva SA, Varfolomeev AF, Chernukha MY, Yurov DS, Vasiliev MM, Kaminskaya AA, et al. Bactericidal effects of non-thermal argon plasma in vitro, in biofilms and in the animal model of infected wounds. J Med Microbiol. 2011; 60(1):75-83.

30. Hong YF, Kang JG, Lee HY, Uhm HS, Moon E, Park YH. Sterilization effect of atmospheric plasma on Escherichia coli and Bacillus subtilis endospores. Lett Appl Microbiol. 2009; 48(1):33-7.

31. Scholtz V, Pazlarova J, Souskova H, Khun J, Julak J. Nonthermal plasma - A tool for decontamination and disinfection. Biotechnol Adv. 2015; 33(6):1108-19.

32. Sladek REJ, Filoche SK, Sissons CH, Stoffels E. Treatment of Streptococcus mutans biofilms with a nonthermal atmospheric plasma. Lett Appl Microbiol. 2007; 45(3):318-23.

33. Canullo L, Micarelli C, Bettazzoni L, Koçi B, Baldissara P. ZirconiaComposite Bonding After Plasma of Argon Treatment. Int J Prosthodont. 2014; 27(3):267-9.

34. Ito $\mathrm{Y}$, Okawa T, Fukumoto T, Tsurumi A, Tatsuta M, Fujii T, et al Influence of atmospheric pressure low-temperature plasma treatment on the shear bond strength between zirconia and resin cement. J Prosthodont Res. 2016; 60(4):289-93.

35. Dong X, Ritts AC, Staller C, Yu Q, Chen M, Wang Y. Evaluation of plasma treatment effects on improving adhesive-dentin bonding by using the same tooth controls and varying cross-sectional surface areas. Eur J Oral Sci. 2013; 121(4):355-62.

36. Kim JH, Han GJ, Kim CK, Oh KH, Chung SN, Chun BH, et al. Promotion of adhesive penetration and resin bond strength to dentin using nonthermal atmospheric pressure plasma. Eur J Oral Sci. 2016; 124(1):89-95.

37. Ritts AC, $\mathrm{Li} \mathrm{H}, \mathrm{Yu} \mathrm{Q}, \mathrm{Xu}$ C, Yao X, Hong L, et al. Dentin surface treatment using a non-thermal argon plasma brush for interfacial bonding improvement in composite restoration. Eur J Oral Sci. 2010; 118(5):510-6.

38. Chen M, Zhang Y, Sky Driver M, Caruso AN, Yu Q, Wang Y. Surface modification of several dental substrates by non-thermal, atmospheric plasma brush. Dent Mater. 2013; 29(8):871-80.

39. Zhang Y, Yu Q, Wang Y. Non-thermal atmospheric plasmas in dental restoration: Improved resin adhesive penetration. J Dent. 2014; 42(8):1033-42.

40. Ayres APA, Hirata R, Fronza BM, Lopes BB, Ambrosano GMB, Giannini M. Effect of Argon Plasma Surface Treatment on Bond Strength of Resin Composite Repair. Oper Dent. 2019; 44(2): 75-82.

41. Ferracane JL. Hygroscopic and hydrolytic effects in dental polymer networks. Dent Mater. 2006; 22(3): 211-22.

42. Heydecke G, Zhang F, Razzoog ME. In vitro color stability of doublelayer veneers after accelerated aging. J Prosthet Dent. 2001; 85(6):551-7.

43. Aguiar TR, Francescantonio MDI, Bedran-russo AK, Giannini M. Inorganic Composition and Filler Particles Morphology of Conventional and Self-Adhesive Resin Cements by SEM / EDX. Microsc Res Tech 2012; 75(10):1348-52

44. Matinlinna JP, Lung CYK, Tsoi JKH. Silane adhesion mechanism in dental applications and surface treatments: A review. Dent Mater. 2018; 34(1):13-28

45. Watanabe I, Nakabayashi N. Measurement methods for adhesion to dentine: the current status in Japan. J Dent. 1994; 22(2):67-72

\section{ABSTRACT}

The objective of this study was to evaluate the effect of nonthermal atmospheric plasma (NTAP), alone or combined with sandblasting or adhesive, and water-storage time on the shear bond strength (SBS) of resin composite repair of resin matrix 
ceramics (RMC). Samples $(14 \times 7 \times 1 \mathrm{~mm})$ of three RMCs, Enamic (VITA Zahnfabrik), Cerasmart (GC Corp.), and Lava Ultimate (3M Oral Care), were prepared and submitted to artificial aging (EQ-UV, Equilam) for $300 \mathrm{~h}$. The following surface treatments were performed: (1- Control) sandblasting + silane + adhesive; (2) NTAP; (3) NTAP + adhesive; (4) sandblasting + NTAP. Resin composite cylinders (Spectra Smart, Dentsply Sirona, 1,5 mm diameter and height) were bonded to the treated surfaces and the SBS was evaluated in a universal testing machine (EZ Test, Shimadzu) after $24 \mathrm{~h}$ or 1 year of water storage, at $37 \mathrm{oC}(\mathrm{n}=10)$. In general, the control treatment obtained the best SBS results, compared to groups treated with NTAP. There was a decrease in SBS after 1 year of water immersion for most groups, including the control treatment for Enamic and Lava Ultimate, while Cerasmart presented no reduction. The treatment with NTAP alone or combined with another treatment, was not capable of increasing the SBS of resin composite repair to RMCs. Control treatment seems to be the best method for repairing CMRs, mainly considering the treatment longevity.

Keywords: CAD-CAM; Dental restoration repair; Shear strength; Non-thermal atmospheric pressure plasma, composite resins, dental porcelain

\section{AUTOR PARA CORRESPONDÊNCIA}

Eduardo Fernandes de Castro

Departamento de Odontologia Restauradora, Faculdade de Odontologia de Piracicaba, Universidade Estadual de Campinas, Av. Limeira, 901 - Bairro Areião, Piracicaba, São Paulo - CEP.: 13414-903

E-mail: eduardofdecastro@hotmail.com

Telefone: +55 (62) 3215-7419 